\title{
The Role of Lymphocyte-To-Monocyte Ratio In Predicting The Severe Post-Calcific Stenotic Aortic Dilatation In A Chinese Case-Control Study
}

\section{Shanghao Chen}

Shandong Provincial Hospital, Shandong University

\section{Zezhong Wu}

Shandong Provincial Hospital affiliated to Shandong First Medical University

\section{Yan Yun}

Qilu Hospital of Shandong University

\section{Hechen Shen}

Shandong Provincial Hospital, Shandong University

\section{Diming Zhao}

Shandong Provincial Hospital, Shandong University

\section{Yanwu Liu}

Shandong Provincial Hospital, Shandong University

\section{Chengwei Zou}

Shandong Provincial Hospital, Shandong University

\section{Haizhou Zhang}

Shandong Provincial Hospital, Shandong University

\section{Zhengjun Wang}

Shandong Provincial Hospital, Shandong University

Xiaochun Ma ( $D$ mxcmxc2008@163.com )

Shandong Provincial Hospital, Shandong University

\section{Research Article}

Keywords: Lymphocyte-to-Monocyte Ratio, Post-Calcific Stenotic Aortic Dilatation, Predict

Posted Date: November 23rd, 2021

DOI: https://doi.org/10.21203/rs.3.rs-1043408/v1

License: (1) (1) This work is licensed under a Creative Commons Attribution 4.0 International License.

Read Full License 


\section{Abstract}

Background: Calcific aortic valve stenosis (CAVS) represents a serious public health threat to elderly patients. Post-calcific stenotic aortic dilatation, a common feature in CAVS patients, might progress into aneurysm and even dissection, potentially lethal consequences of CAVS, and predicts a dismal prognosis. This study sought to testify the role of lymphocyte-to-monocyte ratio (LMR), an inflammatory biomarker, in predicting the severe post-calcific stenotic aortic dilatation in a Chinese case-control study.

Materials and Methods: 208 consecutive patients with CAVS were recruited retrospectively in a Chinese case- control study, from July 1, 2015 to June 31, 2018. The LMR was statistically analyzed for its prognostic value in the severe post-calcific stenotic aortic dilatation.

Results: The LMR was significantly reduced in patients with severe post-calcific stenotic aortic dilatation ( 2.72 vs. $3.53, p=0.002<0.05$ ) compared to patients without severe post- calcific stenotic aortic dilatation. There was an inverse correlation observed between the maximal diameter of ascending aorta and the LMR in the overall patients $(r=-0.217, p=0.002<0.05)$. For severe post-calcific stenotic aortic dilatation, the incidence of high- LMR group was statistically lower than that of low-LMR group (19.7\% vs. 43.9\%, $\mathrm{p}<0.001)$. And maximal diameter of ascending aorta was significantly reduced in the high-LMR group (4.35vs. 4.76, $p=0.003<0.05$ ) compared to low-LMR group. Additionally, the LMR was identified in the multivariate analysis as an independent predictor of severe post-calcific stenotic aortic dilatation (OR $0.743,95 \% \mathrm{Cl}:$ [0.573-0.964], $p=0.025)$.

Conclusions: This study provided the evidence of an inverse correlation between the severe post- calcific stenotic aortic dilatation and LMR. And the LMR is potentially applied as an independent predictor of severe post-calcific stenotic aortic dilatation.

\section{Introduction}

Calcific aortic valve stenosis (CAVS) is the most prevailing heart valve disorder and affects nearly $1.7 \%$ in the population $>65$ years old in developed countries (1). The prevalence has also rapidly elevated in developing countries with the ageing of the population(2). Post-calcific stenotic aortic dilatation, is a common feature in CAVS patients, especially in those with bicuspid aortic valve (BAV) (3). It might progress into aneurysm and even dissection, potentially lethal consequences of CAVS, and predicts a dismal prognosis (4). Thus, post-calcific stenotic aortic dilatation warrants regular medical monitoring and possible early radical surgical intervention (including surgical aortic valve, root and ascending aorta replacement) to prevent catastrophic dissection or rupture. In the management of patients with CAVS, it is necessary to decipher additional risk for post-calcific stenotic aortic dilatation, in an attempt to optimize the assessment of dilatation progression, and clinical decision-making of a combined aorta replacement surgery. However, current data are scarce regarding the determinants and predictors of post-calcific stenotic aortic dilatation. 
The pathophysiology of post-calcific stenotic aortic dilatation remains largely undetermined. Current evidence supported that this entity of aortic dilatation might be due to the unfavorable haemodynamic conditions downstream of stenotic valves, or due to the valve anatomy (BAV and tricuspid aortic valve [TAV]) as well as the intrinsic pathology of aorta (5). Given a pivotal role of inflammation widely recognized in the pathogenesis of CAVS, inflammation might also be linked with the development of postcalcific stenotic aortic dilatation. Of note, several inflammatory diseases as well as inflammatory biomarkers have been correlated with the occurrence of aortic dilatation, suggesting the effects of systemic and localized inflammation on the pathophysiology (6-9).

Lymphocyte-to-monocyte ratio (LMR) is an inflammatory biomarker calculated from peripheral blood count of lymphocytes and monocyte/macrophages. It has recently been emphasized as associated with systemic inflammation and prognosis in various malignancies (10-14). A lower LMR has been demonstrated linked with an increased level of systemic inflammation (15). Here the LMR is hypothesized to function as a practical predictive indicator of severe post-calcific stenotic aortic dilatation. To our best knowledge, such investigation is still missing, focused on the potential relationship of LMR and severe post-calcific stenotic aortic dilatation in a Chinese case-control study.

\section{Material And Methods}

\subsection{Participants and study design}

This study was a single-center retrospective case-control study. It was approved by the Ethics Committee of Shandong Provincial Hospital affiliated to Shandong First Medical University and Shandong University and the approval code for this study is NSFC2019-021. Due to its retrospective design, the written informed consents had to be waived. The study was performed in accordance with the Good Clinical Practice (GCP) and principles of the Declaration of Helsinki (16). 208 patients were consecutively included in the study from the hospital above-mentioned, from July 1, 2015 to June 31, 2018. The time interval of this study was based on its funding which spanned roughly a similar period. All the included patients met the following criteria: (i) older than 18 years; (ii) having a clear diagnosis of CAVS based on the criteria by the American College of Cardiology (ACC) and the American Heart Association (AHA). The subjects were excluded due to the following exclusion criteria: (i) the presence of rheumatic aortic valve stenosis or insufficiency, severe stenosis or insufficiency of mitral or tricuspid valves, endocarditis, coronary artery disease, atrial fibrillation, autoimmune diseases, malignancies and renal, hepatic or hematologic disorders; (ii) whose related data were not sufficient. The recruited patients underwent a comprehensive preoperative evaluation of transthoracic echocardiography (TTE) and aortic CT angiography (CTA). Full blood counts were routinely collected for calculating the LMR by dividing the number of lymphocytes by the number of monocytes.

\subsection{The definition of severe post-calcific stenotic aortic dilatation}


The maximal diameter of ascending aorta was measured by aortic CTA and the severe post-calcific stenotic aortic dilatation was defined as the maximal diameter equal to or greater than $50 \mathrm{~mm}$.

\subsection{The calculation of LMR and grouping by LMR}

The LMR was measured according to the lymphocyte and monocyte counts in the blood routine test. The cut-off LMR was decided by the diagnostic test as described below. The included patients were accordingly grouped as the low-LMR group (with their LMR values less than the cut-off values) and highLMR group (with their LMR values greater than the cut-off values).

\subsection{Statistical analysis}

All statistical analysis was performed using the software SPSS Statistics 25.0. The continuous variables were present in the form of mean \pm standard deviation (SD) if the data conforms to normal distribution, or expressed as median (quartile deviation) if the data inconsistent with the normal distribution. The categorical variables were shown as frequencies (n) with percentages (\%). For analyzing the continuous variables, the Student t-test was used when the normal distribution was conformed. Otherwise, the nonparametric Mann-Whitney U test was applied if a skewed distribution was met. The Chi-square or Fisher's exact test was carried out for analyzing the categorical variables. The cut-off LMR was determined by the diagnostic tests using the receiver operating curve (ROC). The binary logistic regression analysis was employed for the univariable and multivariable analyses. When the efforts were made to construct a multivariable predictive model, all candidate variables derived from the univariable analysis (with a $\mathrm{p}$ value less than 0.1 ) as well as those possible predictive variables were selected. A two-sided $p$ value less than 0.05 was considered statistically significant.

\section{Results}

\subsection{Characteristics of patients}

\subsubsection{Inclusion of patients}

From July 1, 2015 to June 31, 2018, 243 patients were recruited in sequence. 23 subjects were excluded because of the presence of rheumatic aortic valve stenosis (11 cases), severe stenosis or insufficiency of other valves ( 15 cases), endocarditis ( 2 cases), coronary artery disease (8 cases), atrial fibrillation (12 cases), autoimmune disease (2 cases), malignancies and renal, hepatic or hematologic disorders (3 cases); 12 patients excluded whose preoperative and intraoperative data were insufficient (Figure 1).

\subsubsection{Detailed information of patients}

For the patients with severe post-calcific stenotic aortic dilatation, $71.9 \%$ were male patients with a median age of 60.0 (11.0) years. $54.4 \%$ of patients were cigarette smokers, 33.3\% had hypertension and $5.3 \%$ were with type 2 diabetes. $59.6 \%$ of patients had tricuspid aortic valve. The median LVEF was 57.0 (7.5). For the patients without severe post-calcific stenotic aortic dilatation, $62.3 \%$ were male patients with 
a median age of 60.0 (12.0) years. $41.7 \%$ of patients were cigarette smokers, $37.1 \%$ had hypertension and $9.9 \%$ were with type 2 diabetes. $52.3 \%$ of patients had tricuspid aortic valve. The median LVEF was 60.0 (7.0). The detailed information of patients with and without post-calcific stenotic aortic dilatation were summarized in the Table 1. 
Table 1

Clinical Characteristics of Patients with post-calcific stenotic aortic dilatation or not Post-calcific stenotic aortic dilatation Not

P Value

Patient population ( $n$ )

Demographic data

Age (y)

Sex, male (n)

Medical history

Hypertension ( $n$ )

Smoking ( $n)$

Diabetes (n)

LV $(\mathrm{cm})$

LVEF (\%)

Tricuspid aortic valve (n)

Preoperative laboratory tests

Leukocyte $\left(10^{9} / \mathrm{L}\right)$

Neutrophil $\left(10^{9} / \mathrm{L}\right)$

Platelet $\left(10^{9} / \mathrm{L}\right)$

Monocyte $\left(10^{9} / \mathrm{L}\right)$

Lymphocyte $\left(10^{9} / \mathrm{L}\right)$

LMR

LDL-C (mmol/L)

$\mathrm{CRP}(\mathrm{mg} / \mathrm{L})$

MPV (fl)
57

151

60.0(11.0)

$41(71.9 \%)$

19(33.3\%)

$31(54.4 \%)$

$3(5.3 \%)$

6.02(1.74)

$57(7.5)$

$34(59.6 \%)$

6.11(2.06)

$5.89(1.96)$

0.405

$3.48(1.54)$

178(75.5)

$0.59(0.37)$

1.74(0.68)

2.72(1.62)

2.78(1.15)

0.93(0.91)

10.60(1.15)
$60.0(12.0) \quad 0.909$

$94(62.3 \%) \quad 0.174$

63(41.7\%) $\quad 0.094$

$15(9.9 \%) \quad 0.435$

5.45(1.49) 0.011

60(7)

0.006

$79(52.3 \%) \quad 0.321$

$3.49(1.51) \quad 0.896$

$195.5(71.5) \quad 0.183$

$0.50(0.24) \quad 0.006$

1.84(0.68) $\quad 0.675$

3.53(1.75) $\quad 0.002$

$2.80(1.19) \quad 0.843$

0.91(1.62) $\quad 0.388$

10.90(1.25) $\quad 0.329$ 
Abbreviations: LV: left ventricle; LVEF: left ventricular ejection fraction; LMR: Lymphocyte-to-monocyte ratio; LDL-C: Low density lipoprotein cholesterol; CRP: C-reaction protein; MPV: mean platelet volume

NOTE. The categorical variables in the table are presented by the number of cases (with percentage) and the continuous variables are expressed by the median (with interquartile range) or mean (with standard deviation).

$P$ values were the results of unpaired t-test or Mann-Whitney $U$ test for continuous variables, and $\chi 2$ test or Fisher's exact test for categorical variables.

P Value: Compare the patients with and without ascending aorta dilatation.

\subsection{LMR and severe post-calcific stenotic aortic dilatation}

\subsubsection{LMR in patients with severe post-calcific stenotic aortic dilatation or not}

The LMR was significantly reduced in patients with severe post-calcific stenotic aortic dilatation (2.72 vs. $3.53, p=0.002<0.05)$ compared to patients without severe post- calcific stenotic aortic dilatation.

\subsubsection{The correlation of LMR and maximal diameter of ascending aorta}

There was an inverse correlation observed between the maximal diameter of ascending aorta and the LMR in the overall patients $(r=-0.217, p=0.002<0.05)$ (Figure 2).

\subsubsection{Severe post-calcific stenotic aortic dilatation in the high-LMR and low-LMR groups}

The cut-off level of LMR was calculated from a diagnostic test with a ROC curve on the occurrence of severe post-calcific stenotic aortic dilatation, which was 2.72. Accordingly, the subjects were assigned into the high-LMR ( $n=142)$ and low-LMR group $(n=66)$. The detailed information of two groups was summarized in Table 2. For severe post-calcific stenotic aortic dilatation, the incidence of high-LMR group was statistically lower than that of low-LMR group ( $19.7 \%$ vs. $43.9 \%, p<0.001)$. And maximal diameter of ascending aorta was significantly reduced in the high-LMR group $(4.35 \mathrm{vs} .4 .76, \mathrm{p}=0.003<0.05)$ compared to low-LMR group (Figure 3). 
Table 2

Clinical Characteristics of Patients with High-LMR or Low-LMR

\begin{tabular}{|c|c|c|c|}
\hline & High-LMR & Low-LMR & P Value \\
\hline Patient population ( $\mathrm{n}$ ) & 142 & 66 & \\
\hline \multicolumn{4}{|l|}{ Demographic data } \\
\hline Age (y) & $59(13)$ & $63(8.25)$ & 0.005 \\
\hline Sex, male $(n)$ & $86(60.6 \%)$ & $49(74.2 \%)$ & 0.048 \\
\hline \multicolumn{4}{|l|}{ Medical history } \\
\hline Hypertension (n) & $42(29.6 \%)$ & $33(50.0 \%)$ & 0.004 \\
\hline Smoking (n) & $62(43.7 \%)$ & $32(48.5 \%)$ & 0.488 \\
\hline Diabetes (n) & $14(9.9 \%)$ & $4(6.1 \%)$ & 0.372 \\
\hline LV (cm) & $5.41(1.43)$ & $6.29(1.41)$ & $<0.001$ \\
\hline Post-calcific stenotic aortic dilatation (n) & $28(19.7 \%)$ & $29(43.9 \%)$ & $<0.001$ \\
\hline Maximal diameter of the ascending aorta $(\mathrm{cm})$ & $4.35(1.03)$ & $4.76(1.60)$ & 0.003 \\
\hline LVEF (\%) & $60(7)$ & $58(7.5)$ & 0.031 \\
\hline Tricuspid aortic valve(n) & $61(43.0 \%)$ & $52(78.8 \%)$ & $<0.001$ \\
\hline \multicolumn{4}{|l|}{ Preoperative laboratory tests } \\
\hline Leukocyte $\left(10^{9} / \mathrm{L}\right)$ & $5.74(1.93)$ & $6.16(1.90)$ & 0.020 \\
\hline Neutrophil $\left(10^{9} / \mathrm{L}\right)$ & $3.33(1.48)$ & $3.73(1.26)$ & 0.004 \\
\hline Platelet $\left(10^{9} / \mathrm{L}\right)$ & 193(69) & 187(85.25) & 0.818 \\
\hline Monocyte $\left(10^{9} / \mathrm{L}\right)$ & $0.47(0.20)$ & $0.79(0.43)$ & $<0.001$ \\
\hline Lymphocyte (109/L) & $1.88(0.51)$ & $1.54(0.67)$ & $<0.001$ \\
\hline LDL-C (mmol/L) & $2.87(1.18)$ & $2.68(1.12)$ & 0.101 \\
\hline CRP (mg/L) & $0.77(1.43)$ & $1.18(2.07)$ & 0.134 \\
\hline MPV (fl) & $10.90(1.30)$ & $10.60(1.15)$ & 0.287 \\
\hline
\end{tabular}


Abbreviations: LV: left ventricle; LVEF: left ventricular ejection fraction; LMR: Lymphocyte-to-monocyte ratio; LDL-C: Low density lipoprotein cholesterol; CRP: C-reaction protein; MPV: mean platelet volume

NOTE. The categorical variables in the table are presented by the number of cases (with percentage) and the continuous variables are expressed by the median (with interquartile range) or mean (with standard deviation).

$P$ values were the results of unpaired t-test or Mann-Whitney $U$ test for continuous variables, and $\chi 2$ test or Fisher's exact test for categorical variables.

P value: Compare the overall patients with Low-LMR or High-LMR.

\subsection{The LMR predicting the severe post-calcific stenotic aortic dilatation Multivariable analysis of risk} factors for severe post-calcific stenotic aortic dilatation was carried out by including all possible predictive variables (including age, sex, hypertension, diabetes, smoking, LMR, neutrophil, platelet, Creaction protein, low- density lipoprotein cholesterol and tricuspid aortic valve). The results showed that the LMR independently predicted the occurrence of severe post-calcific stenotic aortic dilatation (OR 0.743, 95\% Cl: [0.573-0.964], $p=0.025)$.

\section{Discussion}

In this case-control study, 208 patients were enrolled and the correlation between the LMR and the occurrence of severe post-calcific stenotic aortic dilatation was analyzed. The results presented the evidence of a lower LMR in the patients with severe post- calcific stenotic aortic dilatation compared to the patients without post-calcific stenotic aortic dilatation. And the incidence of severe post-calcific stenotic aortic dilatation in the high-LMR group was statistically lower than that in the low-LMR group. Finally, the LMR was considered as a novel predictor for severe post-calcific stenotic aortic dilatation after adjusting other possible variables.

The severe post-calcific stenotic aortic dilatation is often clinically silent until its catastrophic complication, acute aortic dissection or rupture, occurs(17). Although Davies and colleagues demonstrated that the ascending aorta with a diameter within $35-39 \mathrm{~mm}$ is not associated with aortic rupture or dissection, the risk of rupture or dissection increased dramatically by 27 -fold if the diameter of ascending aorta reaches $60 \mathrm{~mm}$ or more (18). Thus, it is a clinical priority to identify the predictive biomarkers or modifiable risk factors in the prevention and treatment of severe post-calcific stenotic aortic dilatation.

The associated risk factors for post-calcific stenotic aortic dilatation in the previous reports were mainly male gender, bicuspid aortic valve, hypertension and smoking (19-21). Aortic dilatation has been recently reported caused by inflammatory diseases such as infectious aortitis, Takayasu arteritis, and giant cell arteritis $(7,8,22)$. And levels of several inflammatory biomarkers such as C-reactive protein (CRP) and interleukin (IL)- 6 are elevated in patients with thoracic aortic aneurysms, suggesting the effects of systemic inflammation on the pathophysiology (6). Of note, an increased activity of matrix 
metalloproteinases (MMPs) has been described in the media of thoracic aortic aneurysm, a characteristic of localized inflammation $(9,23)$. Recent evidence from basic research has also suggested the role of several canonical inflammatory signaling pathways, AP-1 and the ERK $1 / 2$ signaling pathway, in contributing to the inflammation in post-calcific stenotic aortic dilatation (24). However, the detailed mechanisms underlying the inflammatory reaction in post-calcific stenotic aortic dilatation is to still a large extent undetermined. And identifying feasible inflammatory biomarkers will be constructive in predicting the high-risk patients and supportive in early diagnosis and intervention.

LMR is calculated from the exact amounts of lymphocyte and monocyte of peripheral blood and has been uncovered as an independent predictive indicator of clinical outcomes in various cancers(10-14). As an inflammation-related indicator, a lower LMR appears to be associated with decreased survival and increased recurrence in malignancies(15). Our findings demonstrated that the LMR is significantly lower in the patients with severe post-calcific stenotic aortic dilatation compared to the patients without postcalcific stenotic aortic dilatation. And an inverse correlation was observed between the maximal diameter of ascending aorta and the LMR. It indicated that as the maximal diameter of ascending aorta develops from the normal status to dilatation, the LMR might reduce proportionally. Thus, the patients with CAVS having a lower LMR could be potentially categorized into the high-risk population of post-calcific stenotic aortic dilatation. With the aid of risk stratification of post-calcific stenotic aortic dilatation by LMR, early identification of patients at high risk might be realized, which would lead to the close monitoring and early initiation of efficient preventive and therapeutic strategies. And a more accurate cut-off LMR might also be used in early diagnosis of post-calcific stenotic aortic dilatation if more prospective studies with larger sample sizes in multiple ethnicities are available to confirm it.

\section{Limitations}

Several limitations were present and special attention should be paid to interpret the results in this study. First of all, the study design, a single-center retrospective observational study, inevitably introduced a source of potential bias. The second limitation lies in a small simple size. Third, some confounding variables, such as other inflammatory markers, might influence the role of LMR in post-calcific stenotic aortic dilatation. Fourth, the association of LMR and mid- and long-term endpoints was not investigated. Fifth, the case-control design of this study did not evaluate the causal relationships between LMR and post-calcific stenotic aortic dilatation.

\section{Conclusions}

The LMR is inversely correlated with the incidence and severity of post-calcific stenotic aortic dilatation and might independently predict the occurrence of post- calcific stenotic aortic dilatation. Further largescale case-control studies are needed to confirm these results in the future.

\section{List Of Abbreviations}




\begin{tabular}{|l|l|}
\hline Term & Abbreviations \\
\hline Calcific Aortic Valve Stenosis & CAVS \\
\hline Bicuspid Aortic Valve & BAV \\
\hline Tricuspid Aortic Valve & TAV \\
\hline Lymphocyte-to-monocyte ratio & LMR \\
\hline Good Clinical Practice & GCP \\
\hline American College of Cardiology & ACC \\
\hline American Heart Association & AHA \\
\hline Transthoracic Echocardiography & TTE \\
\hline CT Angiography & CTA \\
\hline Standard Deviation & SD \\
\hline Receiver Operating Curve & ROC \\
\hline C-reactive Protein & CRP \\
\hline Matrix Metalloproteinases & MMPs \\
\hline
\end{tabular}

\section{Declarations}

Ethics approval and consent to participate:

This study was approved by the Ethics Committee of Shandong Provincial Hospital affiliated to Shandong First Medical University and Shandong University and the approval code for this study is NSFC2019-021.

Consent for publication:

Not applicable.

Availability of data and materials:

The datasets used and analysed during the current study are available from the corresponding author on reasonable request. 
Competing interests:

The authors declare that they have no competing interests.

\section{Funding:}

The current work was supported by grants from the National Nature Science Foundation of China (81800255 to MX) and Natural Science Foundation of Shandong Province (ZR2018BH002 to MX and ZR2020MH044 to ZC).

Authors' contributions:

SC and ZW analyzed and interpreted the patient data regarding the severe post-calcific stenotic aortic dilatation. $\mathrm{XM}$ and $\mathrm{YY}$ were major contributor in writing the manuscript. All authors read and approved the final manuscript.

Acknowledgements:

Not applicable.

Disclosure Statement: we have no conflict of interest to declare in the manuscript.

\section{References}

1. Lindman BR, Clavel MA, Mathieu P, lung B, Lancellotti P, Otto CM, et al. Calcific aortic stenosis. Nat Rev Dis Primers. 2016;2:16006.

2. Peeters F, Meex SJR, Dweck MR, Aikawa E, Crijns H, Schurgers LJ, et al. Calcific aortic valve stenosis: hard disease in the heart: A biomolecular approach towards diagnosis and treatment. Eur Heart $\mathrm{J}$. 2018;39(28):2618-24.

3. Wilton E, Jahangiri M. Post-stenotic aortic dilatation. J Cardiothorac Surg. 2006;1:7.

4. Zhang X, Luo M, Fang K, Li J, Peng Y, Zheng L, et al. Analysis of the formation mechanism and occurrence possibility of Post-Stenotic Dilatation of the aorta by CFD approach. Comput Methods Programs Biomed. 2020;194:105522.

5. Edlin J, Youssefı P, Bilkhu R, Figueroa CA, Morgan R, Nowell J, et al. Haemodynamic assessment of bicuspid aortic valve aortopathy: a systematic review of the current literature. Eur $\mathrm{J}$ Cardiothorac Surg. 2019;55(4):610-7.

6. Artemiou P, Charokopos N, Rouska E, Sabol F, Chrysogonidis I, Tsavdaridou V, et al. C- reactive protein/interleukin- 6 ratio as marker of the size of the uncomplicated thoracic aortic aneurysms. Interact Cardiovasc Thorac Surg. 2012;15(5):871-7.

7. Muratore F, Salvarani C. Aortic dilatation in a patient with Takayasu arteritis treated with tocilizumab. Ann Rheum Dis. 2021;80(7):e121. 
8. Nakayama M, Fuse K, Sato M, Okabe M, Misumi S, Yamashina A, et al. Infectious aortitis caused by Salmonella Dublin followed by aneurysmal dilatation of the abdominal aorta. Intern Med. 2012;51(20):2909-11.

9. Shen M, Lee J, Basu R, Sakamuri SS, Wang X, Fan D, et al. Divergent roles of matrix metalloproteinase 2 in pathogenesis of thoracic aortic aneurysm. Arterioscler Thromb Vasc Biol. 2015;35(4):888-98.

10. Fu X, Li T, Dai Y, Li J. Preoperative systemic inflammation score (SIS) is superior to neutrophil to lymphocyte ratio (NLR) as a predicting indicator in patients with esophageal squamous cell carcinoma. BMC Cancer. 2019;19(1):721.

11. Lochowski M, Lochowska B, Zawadzka I, Cieslik-Wolski B, Kozik D, Kozak J. Prognostic value of neutrophil-to-lymphocyte, platelet-to-lymphocyte and lymphocyte-to-monocyte ratio ratios in patients operated on due to non-small cell lung cancer. J Thorac Dis. 2019;11(8):3377-84.

12. Lu C, Zhou L, Ouyang J, Yang H. Prognostic value of lymphocyte-to-monocyte ratio in ovarian cancer: A meta-analysis. Medicine (Baltimore). 2019;98(24):e15876.

13. Tham T, Wotman M, Chung C, Ahn S, Dupuis H, Gliagias V, et al. Systemic immune response in squamous cell carcinoma of the head and neck: a comparative concordance index analysis. Eur Arch Otorhinolaryngol. 2019;276(10):2913-22.

14. Wang Y, Huang D, Xu WY, Wang YW, Che GW. Prognostic Value of Pretreatment Lymphocyte-toMonocyte Ratio in Non-Small Cell Lung Cancer: A Meta-Analysis. Oncol Res Treat. 2019;42(10):52331.

15. Dupre A, Malik HZ. Inflammation and cancer: What a surgical oncologist should know. Eur J Surg Oncol. 2018;44(5):566-70.

16. World Medical A. World Medical Association Declaration of Helsinki: ethical principles for medical research involving human subjects. JAMA. 2013;310(20):2191-4.

17. Bhave NM, Eagle KA. "Much More Than a Tube": The Aneurysmal Ascending Aorta as a Dynamic Entity. JACC Cardiovasc Imaging. 2019;12(6):1030-1.

18. Davies RR, Goldstein LJ, Coady MA, Tittle SL, Rizzo JA, Kopf GS, et al. Yearly rupture or dissection rates for thoracic aortic aneurysms: simple prediction based on size. Ann Thorac Surg. 2002;73(1):17-27; discussion -8.

19. Johnston KW, Rutherford RB, Tilson MD, Shah DM, Hollier L, Stanley JC. Suggested standards for reporting on arterial aneurysms. Subcommittee on Reporting Standards for Arterial Aneurysms, Ad Hoc Committee on Reporting Standards, Society for Vascular Surgery and North American Chapter, International Society for Cardiovascular Surgery. J Vasc Surg. 1991;13(3):452-8.

20. Rogers IS, Massaro JM, Truong QA, Mahabadi AA, Kriegel MF, Fox CS, et al. Distribution, determinants, and normal reference values of thoracic and abdominal aortic diameters by computed tomography (from the Framingham Heart Study). Am J Cardiol. 2013;111(10):1510-6.

21. Roman MJ, Devereux RB, Kramer-Fox R, O'Loughlin J. Two-dimensional echocardiographic aortic root dimensions in normal children and adults. Am J Cardiol. 1989;64(8):507-12. 
22. Jud P, Verheyen N, Dejaco C, Haas E, Szolar D, Meinitzer A, et al. Prevalence and prognostic factors for aortic dilatation in giant cell arteritis - a longitudinal study. Semin Arthritis Rheum. 2020.

23. Li T, Jiang B, Li X, Sun HY, Li XT, Jing JJ, et al. Serum matrix metalloproteinase-9 is a valuable biomarker for identification of abdominal and thoracic aortic aneurysm: a case-control study. BMC Cardiovasc Disord. 2018;18(1):202.

24. Tobin SW, Alibhai FJ, Lee MM, Yeganeh A, Wu J, Li SH, et al. Novel mediators of aneurysm progression in bicuspid aortic valve disease. J Mol Cell Cardiol. 2019;132:71-83.

\section{Figures}

243 patients were successively included in the study from July 1, 2015 to June 31, 2018.

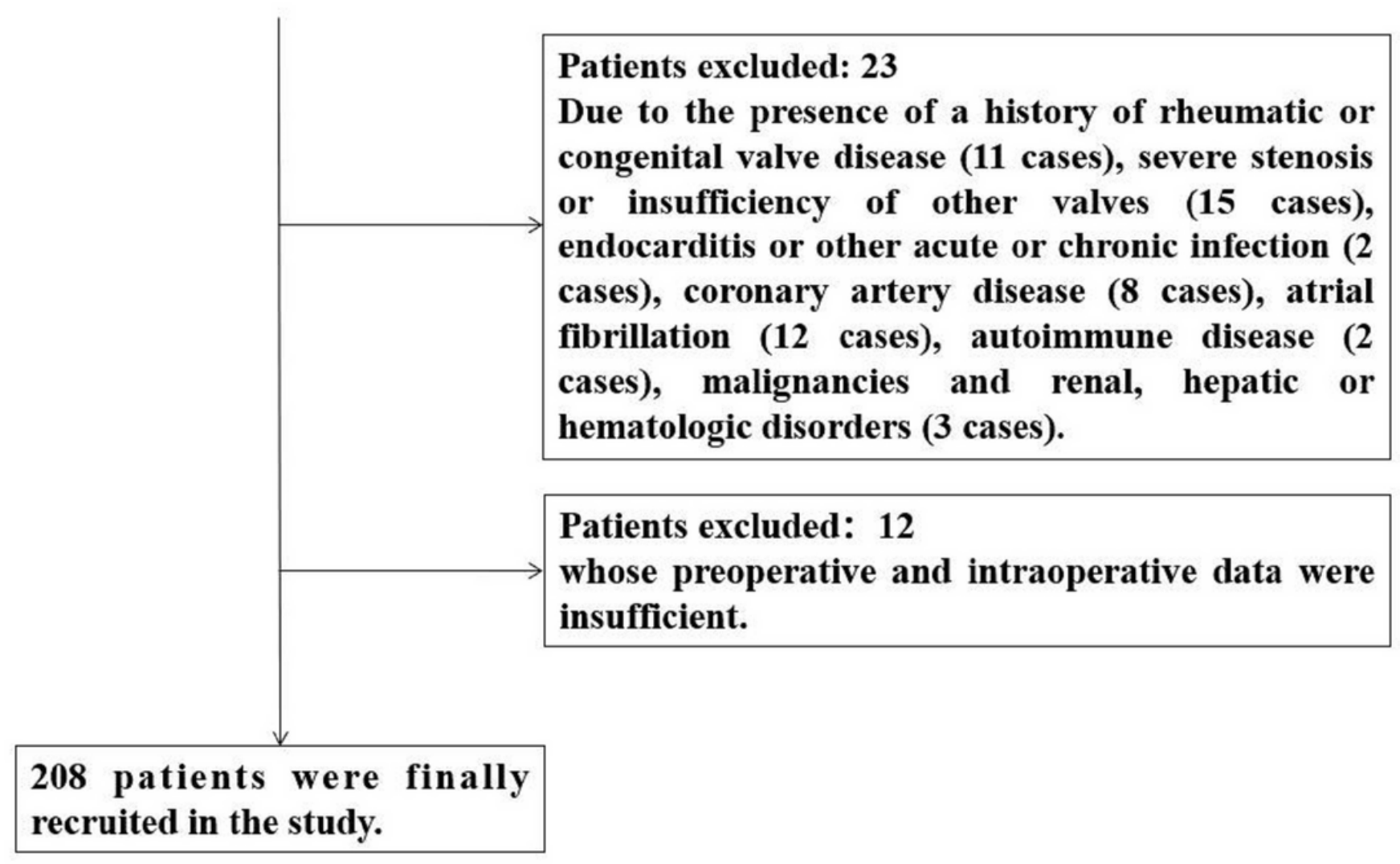

Figure 1

Flow diagram of exclusion and enrollment of study patients describes the exclusion and enrollment of study patients. 


\section{Overall patients}

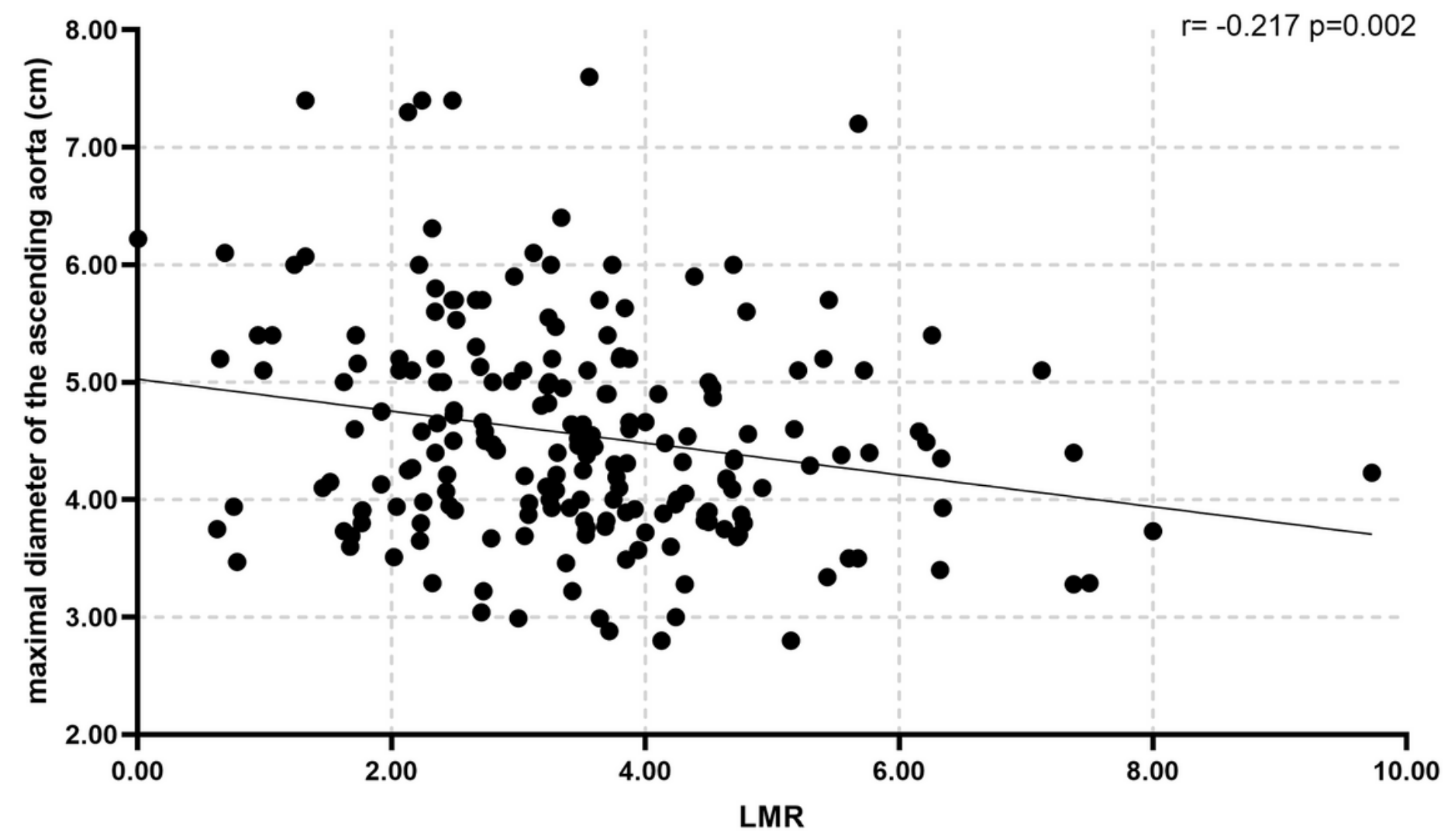

Figure 2

The correlation of LMR and maximal diameter of ascending aorta shows the inverse correlation between the maximal diameter of ascending aorta and the LMR in the overall patients $(r=-0.217, p=0.002<0.05)$. LMR, Lymphocyte-to-Monocyte Ratio. 


\section{Overall Patients}

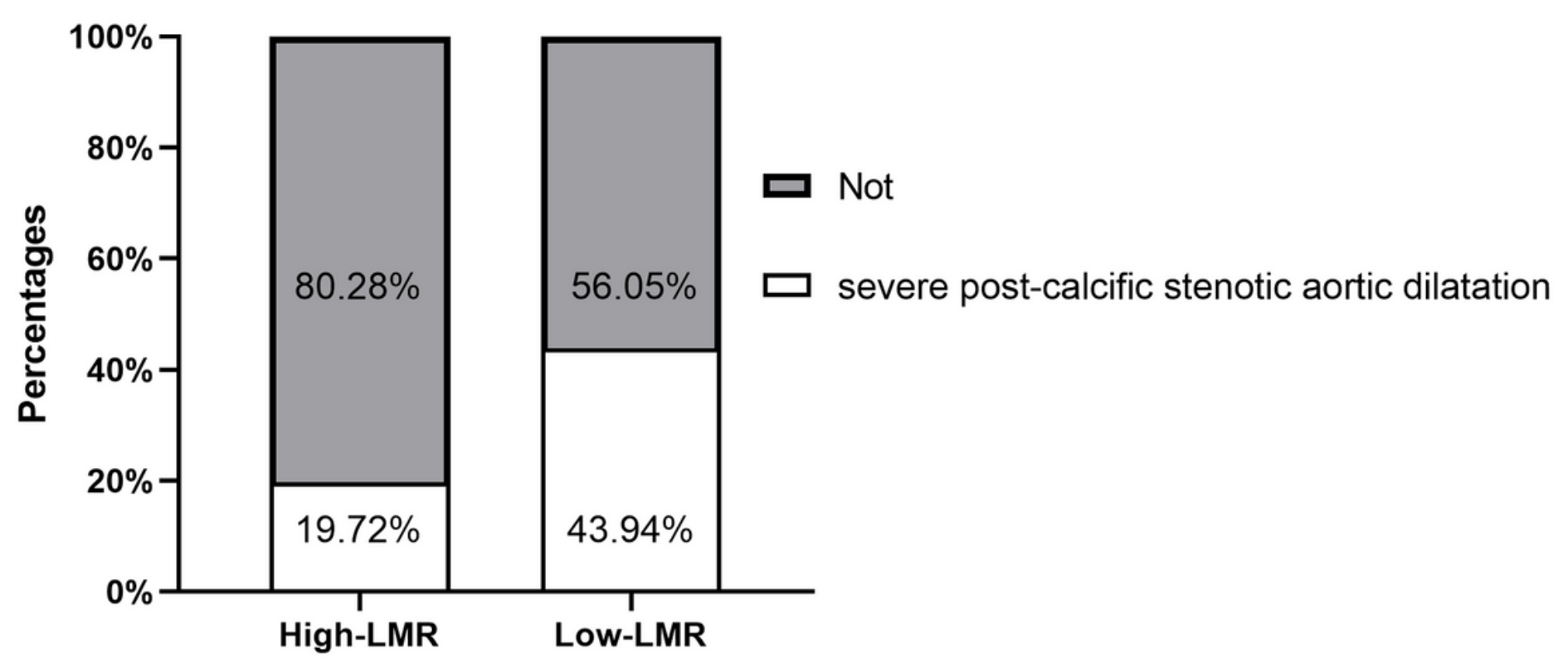

Figure 3

The incidence of Severe post-calcific stenotic aortic dilatation in the high- LMR and low-LMR groups depicts the incidence of Severe post-calcific stenotic aortic dilatation in the high-LMR and low-LMR groups. LMR, Lymphocyte-to-Monocyte Ratio. 